\title{
Rapamycin induces autophagy and increases heat tolerance in Drosophila melanogaster
}

\author{
Quentin Willot ${ }^{1 *}$, Andre du Toit ${ }^{2}$, Ben Loos ${ }^{2}$, John S. Terblanche ${ }^{1}$
}

1. Center for Invasion Biology, Department of Conservation Ecology \& Entomology, Stellenbosch

University, Stellenbosch, South Africa

2. Department of Physiological Sciences, Stellenbosch University, Stellenbosch, South Africa

*Corresponding author: Quentin Willot (qwillot@sun.ac.za), Aarhus University, Department of Zoophysiology, C.F. Mollers Allé 3, Building 1130, 8000 Aarhus C, Denmark 


\section{Abstract}

Autophagy is a physiological process that facilitates the recycling of intracellular cytosolic components as a response to diverse stressful conditions. By increasing the turnover of damaged structures and clearance of long-lived and larger protein aggregates, the induction of autophagy increases tolerance to abiotic stress in a range of organisms. However, the contribution of this process to heat-tolerance of insect models remains poorly studied to date. Here, we report that rapamycin exposure in Drosophila melanogaster induces autophagy in flies, which in turn correlates with an increase in heat tolerance and quicker recovery from heat-coma. This confirms the potentially important role of the autophagic process in heat tolerance mechanisms in this organism, opening the path to further characterization of its relationship to thermal acclimation and molecular level processes related to stress.

\section{Keywords}

Autophagy, rapamycin, heat-tolerance, insects 


\section{Introduction}

Exploring molecular and physiological mechanisms leading to thermal acclimation and increased heat-tolerance in ectotherms has been an intense subject of research (GonzalesTokman et al., 2020; Somero, 2020). Perhaps best studied in organisms such as Drosophila melanogaster and Caenorhabditis elegans, the cellular heat-shock response (HSR) is central in increasing ectotherm's thermal limits (Lindquist, 1985; Feder and Hoffmann 1999; Richter et al., 2010). The HSR entails that macromolecular damage resulting from heat-induced proteotoxic stress triggers a general response under the control of the transcription factor HSF-1 (Pirkkala et al., 2001). This leads to downstream expression of heat-shock proteins, but also opens a range of molecular toolboxes to further increase an organism's resilience to stress (Richter et al., 2010).

Macroautophagy (hereafter referred to as autophagy) is one of these protective tools. In essence, autophagy is a degradation pathway that facilitates the recycling of damaged proteins and larger cytosolic components in response to a multitude of stressors (Mizhushima 2007; Kroemer et al.,, 2010; Maruzs et al., 2019). In Drosophila melanogaster, autophagy induction is under the control of the Target of Rapamycin (TOR) pathway (Zhang et al., 2000; Wullschleger et al., 2006; Maruzs et al., 2019). In response to stress, or exposure to rapamycin, inhibition of TOR has been linked to the upregulation of autophagy and downregulation of the synthesis of proteins that are not involved in the stress response, at the expense of cellular proliferation (Wullschleger et al., 2006). This contributes to the maintain of proteostasis, and autophagy has repeatedly been linked with enhanced tolerance to starvation, desiccation, heavy metals, toxic and oxidative damages in a variety of organisms, including insects (Moore et a., 2006; Bjedov et al., 2009, Minois et al., 2012; Teets and Denlinger, 2013; Mizunoe et al., 2018; Zhang et al., 2019; Babczyńska et al., 2020, Valko et al., 2021). In the specific case of heat-stress, it has been demonstrated that activation of HSF-1 induces autophagy in multiple tissues in the ectothermic nematode Caenorhabditis elegans, which reduces protein denaturation and increases survival (Kumsta et al., 2017). Furthermore, rapamycin treatments have previously been shown to increase heat-tolerance in D. melanogaster (Rhode, 2021). Taken together, this provides strong circumstantial evidences for the upregulation of autophagy to be another pivotal mechanism to increase thermal tolerance in insects.

In this work, we thus report that a rapamycin enriched diet does indeed induce autophagy in the flies' gut, and confirm that this is secondary linked to an increase in heattolerance and recovery from heat-coma in $D$. melanogaster adults. We thus further bridge the gap between the induction of autophagy and the phenotypical heat-tolerance observed as a result of rapamycin-treatment in flies. 


\section{Methods}

\subsection{Fly Stocks and rearing}

Wild Drosophila flies were bait-trapped in April 2019 around the Stellenbosch University campus, Western Cape, South Africa. Single female flies were isolated into $250 \mathrm{ml}$ rearing flask supplied with $50 \mathrm{ml}$ of Bloomington cornmeal diet (Lewis, 1960) and left to lay eggs for one day. Between 2 and 5 of the first emerging adults were used for species determination following the key of Markow and Grady (2005). Stocks were kept at a density of 100 flies per flask, at a constant $25^{\circ} \mathrm{C}$ and a $12: 12$ light-dark cycle until experiments.

\subsection{Rapamycin Treatment}

For larval developmental assays, rapamycin (LC Laboratories) was dissolved in molecular biology grade absolute ethanol (Sigma-Alldricht) and added to the Bloomington cornmeal diet (Lewis, 1960) at an concentrations known to induce systemic effects in flies (50 or $200 \mu \mathrm{M}$, Bjedov et al., 2009). For control $(0 \mu \mathrm{M})$, ethanol alone was added. For heatknockdown and recovery assays in adults, rapamycin was dissolved in ethanol and mixed with liquid food ( $5 \%$ yeast extract, $5 \%$ sucrose, $1 \%$ ethanol) to reach the appropriate concentration of rapamycin (50 or $200 \mu \mathrm{M})$. For the control group $(0 \mu \mathrm{M})$, ethanol alone was added. Blue food commercial dye (Brilliant blue FCF) was used to visually confirm feeding events in adults. 8 days old flies were left to feed on this diet for 2 days prior to experiments.

\subsection{Developmental assays}

Single 10 day old female flies were transferred into new vials containing the Bloomington cornmeal diet (Lewis, 1960), supplemented with 0, 50 or $200 \mu \mathrm{M}$ rapamycin, and left to lay eggs for $24 \mathrm{~h}$. Development of larvae in the medium at $23^{\circ} \mathrm{C}$ was monitored daily, and number of pupations and eclosions carefully recorded.

\subsection{Heat-knockdown and recovery assays}

For heat-knockdown experiments at $37^{\circ} \mathrm{C}$, groups of 10 flies exposed to either 0,50 or 200 $\mu \mathrm{M}$ rapamycin for 2 days were isolated into glass vials containing a moist cotton ball. Vials were then submerged in a programmable water bath (GD120 series, Grant Instrument Ltd) and the temperature inside the vials was monitored using $0.075 \mathrm{~mm}$ diameter thermocouples (Type T Thermocouple (Copper/Constantan), OMEGA Engineering, CT, USA) connected to a digital thermometer (Fluke 52-II Dual Input Digital Thermometer, WA, USA). Flies were exposed to a constant $37^{\circ} \mathrm{C}$ and checked every 15 minutes for loss of righting response, defined as the onset of muscle coordination when flies would fall on their back after a gentle vial shake, keep in a curled-up position, and display total absence of movements for more 
than 15 consecutive minutes. Between 115 and 127 flies were used for each experimental condition to extract knockdown curves at $37^{\circ} \mathrm{C}$. For heat-knockdown experiments at $41^{\circ} \mathrm{C}$, because the onset of muscle coordination would occur much faster, single flies were isolated into a glass vials, exposed to $41^{\circ} \mathrm{C}$, and monitored continuously. As soon as the loss of righting response occurred, each heat knocked-down fly was immediately placed back at room temperature $\left(22^{\circ} \mathrm{C}\right)$ and monitored until recovery, defined as the time at which a fly could stand on its legs again, without stumbling (lack of coordinated movement) or falling over from external stimulation (gentle vial shaking). Between 45 and 51 flies were used to reconstruct $41^{\circ} \mathrm{C}$ knockdown and recovery curves as a function of time. Times to HKD at $41^{\circ} \mathrm{C}$ and times to recovery were finally plotted against each other and the dataset screened for potential correlations.

\subsection{Dissection, staining and confocal microscopy}

To visualize the induction of autophagy in $D$. melanogaster, the gut of control or rapamycintreated flies were dissected out in $4^{\circ} \mathrm{C}$ chilled PBS and then stained in a $10 \mu \mathrm{M}$ Lysotracker Red: $1 \mu \mathrm{g} / \mathrm{ml}$ Hoechst solution (LysoTrackerTM Red DND-99, Hoechst 33342 Solution, Thermofisher Scientific) for 3 minutes. Each preparation was washed three times with cold PBS, fixed overnight in $4 \%$ PFA at $4^{\circ} \mathrm{C}$ and mounted within the next 2 hours. Imaging was performed using a Zeiss LSM 780 ELYRA P.S.1 confocal microscope using a 20x objective. In each case, the midgut region between the proventriculus and Malpighian tubules were acquired. Laser power, digital gain and optical settings were kept constant between the images. Images were then binarized using a set threshold and pixel of high values quantified with the ImageJ software 1.50 (Schneider et al., 2012). The ratio of positive pixels per counting area was pooled for each individual and presented as an average made over between 7 and 13 individuals for each condition.

\subsection{Statistical analysis}

Kruskal-Wallis tests, Log-rank tests analyses of Kaplan-Meier survival and development curves as well as linear regressions were performed using GraphPad Prism 6.01.

\section{Results}

\subsection{Rapamycin feeding induces autophagy in the Drosophila gut}

Both larvae and adult flies were fed control or rapamycin supplemented food at 50 and $200 \mu \mathrm{M}$, after which their guts were dissected and stained with Lysotracker prior to imaging. On average, there was a significant increase in the number of Lysotracker-positive lysosomes and autolysosomes observed in the midgut after rapamycin treatment in all life stage, indicative of autophagy induction (Fig.1 A, B). Quantification of the average number of 
pixels above intensity threshold in midgut areas of flies was indicative of a dose-dependent response as a function of rapamycin concentration in food (Fig. 1C, Kruskal-Wallis one-way analysis of variance followed by Dunn's multiple comparisons post hoc test, $p<0.01$ ).

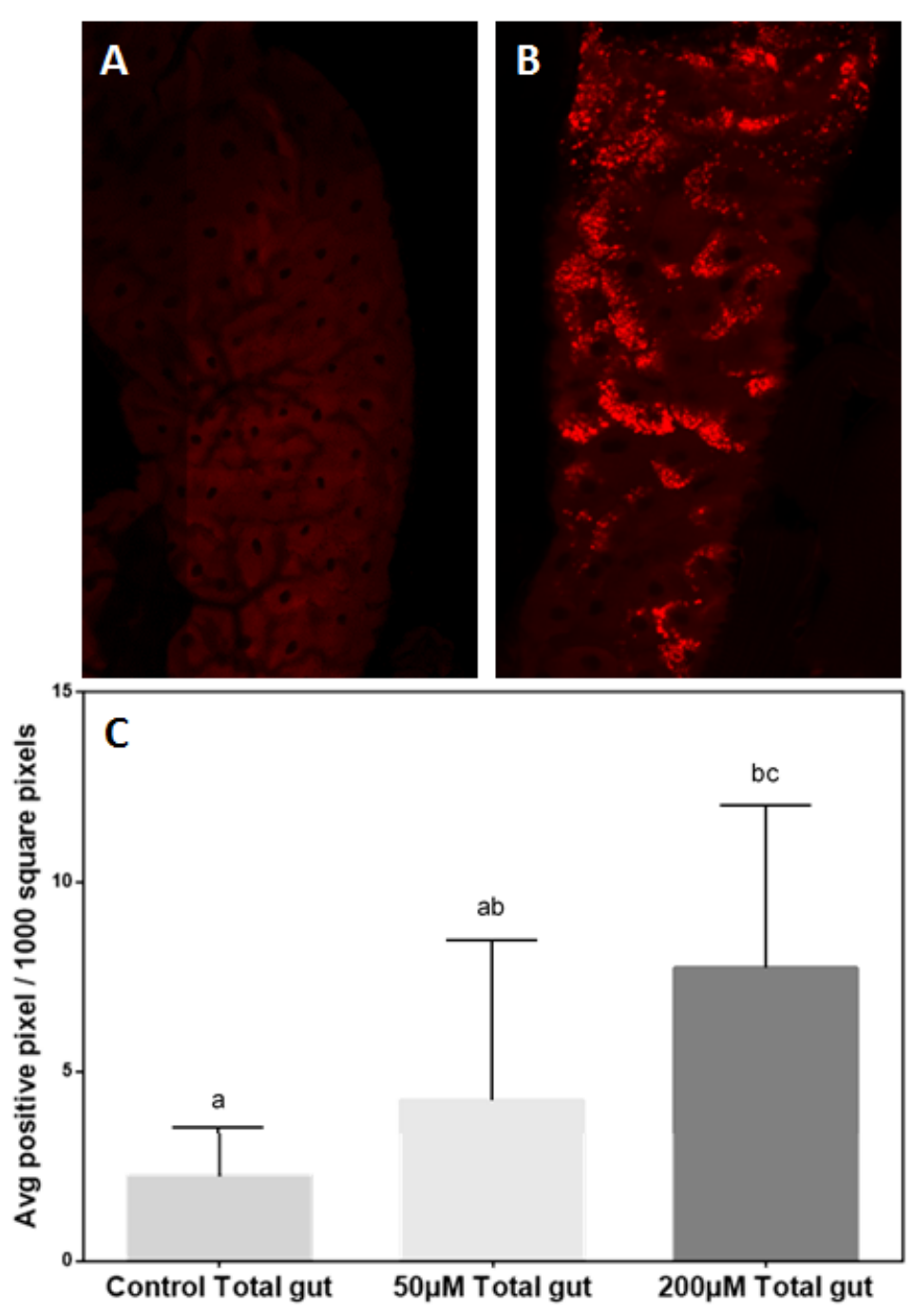

Figure 1. Representative confocal fluorescence images of adult Drosophila midguts stained with LysoTracker Red (scale bar is $50 \mu \mathrm{M}$ ). A. Midgut of control flies. B. Midgut of rapamycin treated flies (200 $\mu \mathrm{M})$. C. Average number of pixels above intensity threshold over midgut images of 7 to 13 individuals per conditions. Different lowercase letters indicate significant differences between treatments (KruskalWallis one-way analysis of variance followed by Dunn's multiple comparisons post hoc test, $\mathrm{P}<0.01)$.

\subsection{Rapamycin feeding delays Drosophila larval development}

Delayed larval development as a result of TOR inhibition is a documented effect of rapamycin exposure in D. melanogaster (Zhang et al., 2000). We thus performed larval growth assays in order to confirm that, in our experimental setup, a rapamycin-enriched diet would indeed induces the expected systemic effects associated with TOR inhibition, in addition to autophagy induction. Larvae were left to develop in Bloomington cornmeal diet (Lewis, 1960) supplemented with either 0 (control), 50 or $200 \mu \mathrm{M}$ of Rapamycin. The number of pupations and imagoes hatching was recorded as a function time at $23^{\circ} \mathrm{C} .50 \mu \mathrm{M}$ 
rapamycin exposure significantly delayed the onset of first larval pupations by about 4 days and hatching by 6 days (Fig.2; Log-ranked test, $p<0.001$ ). Survival from first instar to imago was estimated at $98 \%$ in control conditions ( $\mathrm{N}=453)$ and dropped to $36 \%$ with $50 \mu \mathrm{M}$ supplemented rapamycin food $(\mathrm{N}=129)$. No pupation event was observed at $200 \mu \mathrm{M}$ (all larvae remained either in second or third instars until mortality reached $100 \%$ after 20 days).

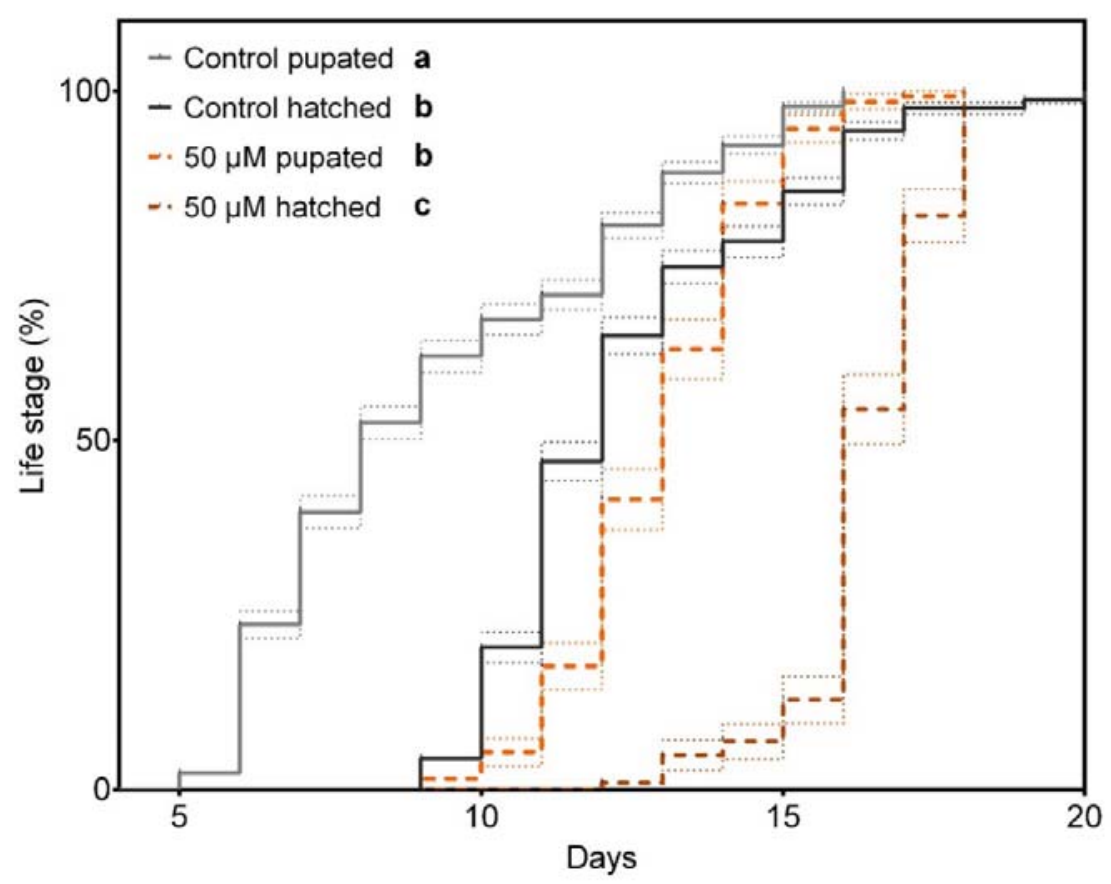

Figure 2. Number of Drosophila melanogaster pupation and hatching events as a function of time at $23^{\circ} \mathrm{C}$, for larvae fed either control or a $50 \mu \mathrm{M}$ rapamycin supplemented diet. Rapamycin exposure delays both the onset of pupation and hatching by several days. Different lowercase letters indicate significant differences between treatments (Log-rank tests, $\mathrm{P}<0.001)$. 


\subsection{Rapamycin treatment delays time to knockdown in flies at $37^{\circ} \mathrm{C}$}

Eight-day old adult flies were fed a rapamycin-enriched diet at either 0 (control), 50 or 200 $\mu \mathrm{M}$ for two days and then subsequently exposed to a static $37^{\circ} \mathrm{C}$ stress to induce a timedependent heat knockdown. The number of knocked down flies as a function of time was recorded every 15 minutes (Fig. 3). Rapamycin exposure delayed the time for flies to enter knockdown (Fig. 3, Log-ranked test, $\mathrm{p}<0.001$ ). $50 \mu \mathrm{M}$ rapamycin exposure in food was already sufficient to induce a plateau response.

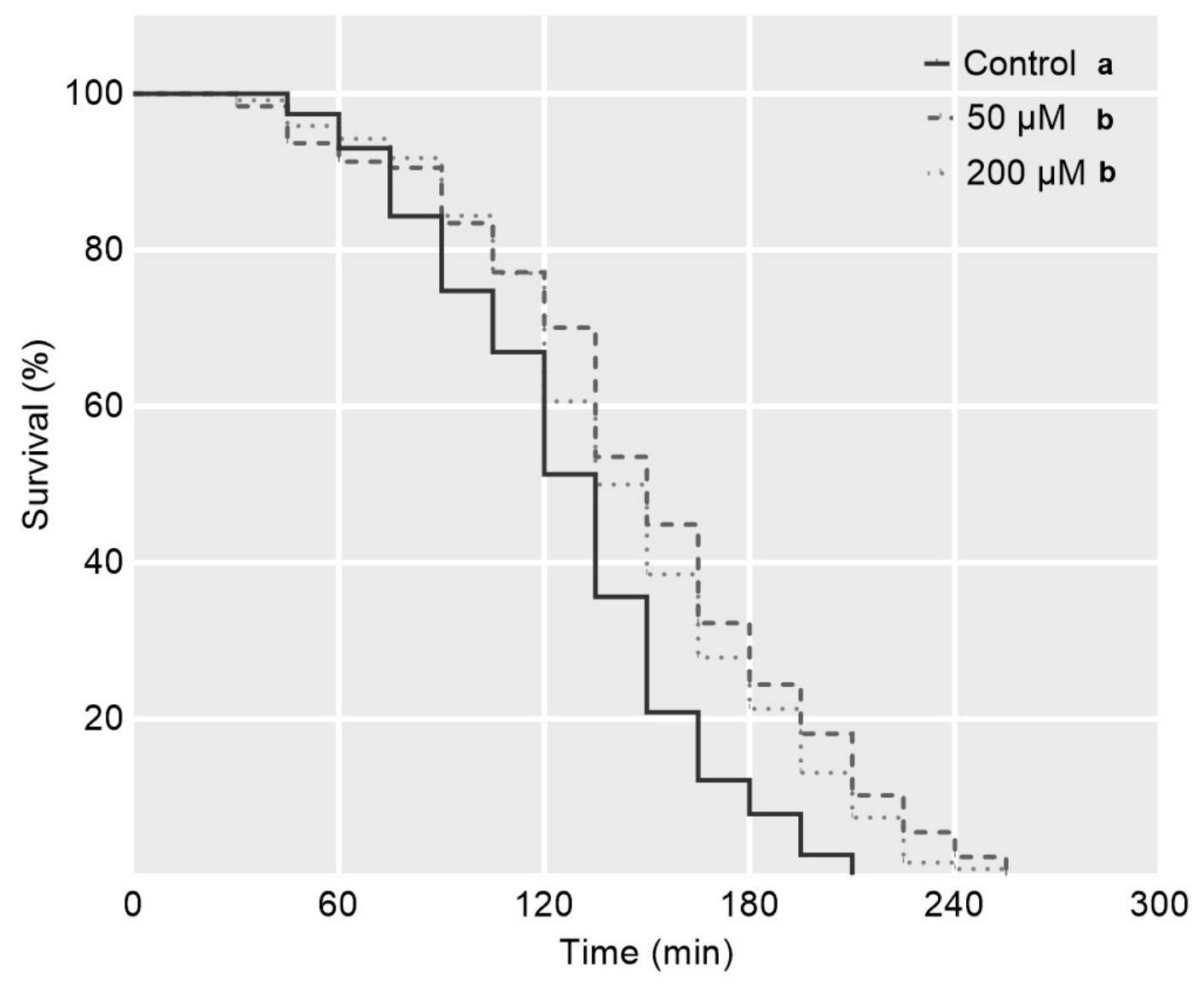

Figure 3. Survival of Drosophila melanogaster flies that were exposed to a rapamycinenriched diet as a function of time at $37^{\circ} \mathrm{C}$. Rapamycin exposure delayed time-to knockdown in flies, and $50 \mu \mathrm{M}$ exposure was sufficient to induce a plateau response. Different lowercase letters indicate significant differences between treatments (Log-rank tests, $\mathrm{P}<0.001$ ).

\subsection{Rapamycin treatment decreases time to recovery post knockdown}


Eight-day old adult flies were fed a rapamycin-enriched diet at either 0 (control), 50 or 200 $\mu \mathrm{M}$ for two days and subsequently exposed to a constant $41^{\circ} \mathrm{C}$ stress to induce a fast heatknockdown. Flies were monitored continuously and put back at room temperature $\left(23^{\circ} \mathrm{C}\right)$ for recovery after the loss of muscle coordination. The impact of rapamycin exposure on fast knockdown and its linked recoveries are presented in Fig. 4. For knockdowns at $41^{\circ} \mathrm{C}$, no effect of rapamycin exposure in delaying time to knockdown was observed (Fig. 4A). However, rapamycin exposure reduced the time to recovery post knockdown in flies (Fig. 4B, Log-ranked test, $\mathrm{p}<0.001)$. Finally, for each individual fly, time to knockdown at $41^{\circ} \mathrm{C}$ was plotted against its time to recovery, and significant regressions were screened both as a function of rapamycin exposure (Fig. 5A) and on the global dataset (Fig. 5B; Table 1). Only a weak correlation between time to knockdown and recovery was found for flies fed with $50 \mu \mathrm{M}$ rapamycin $\left(R^{2}=0.22, p<0.001\right.$, Fig. $\left.5 A\right)$ and on the global dataset $\left(R^{2}=0.05, p<0.01\right.$; Fig. 5B). No significant correlations were recorded for other conditions (Table 1).
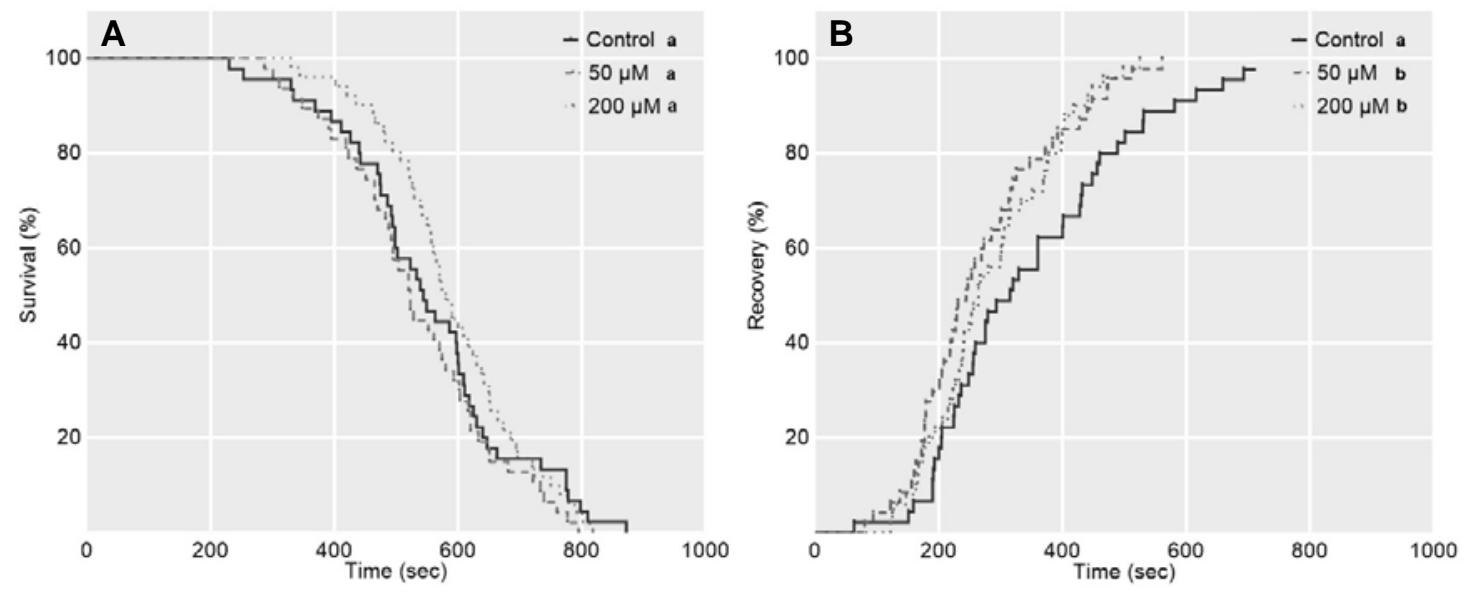

Figure 4. Heat knockdown and recovery curves of $D$. melanogaster flies that were exposed to a rapamycin-enriched diet as a function of time at $41^{\circ} \mathrm{C}$. Fig. 4A. Rapamycin exposure did not delay time to heat knockdown in flies at $41^{\circ} \mathrm{C}$. Fig. 4B. Rapamycin exposure decreased time to recovery from heat knockdown of flies. Different lowercase letters indicate significant differences between treatments (Log-rank tests, $\mathrm{P}<0.001$ ). 

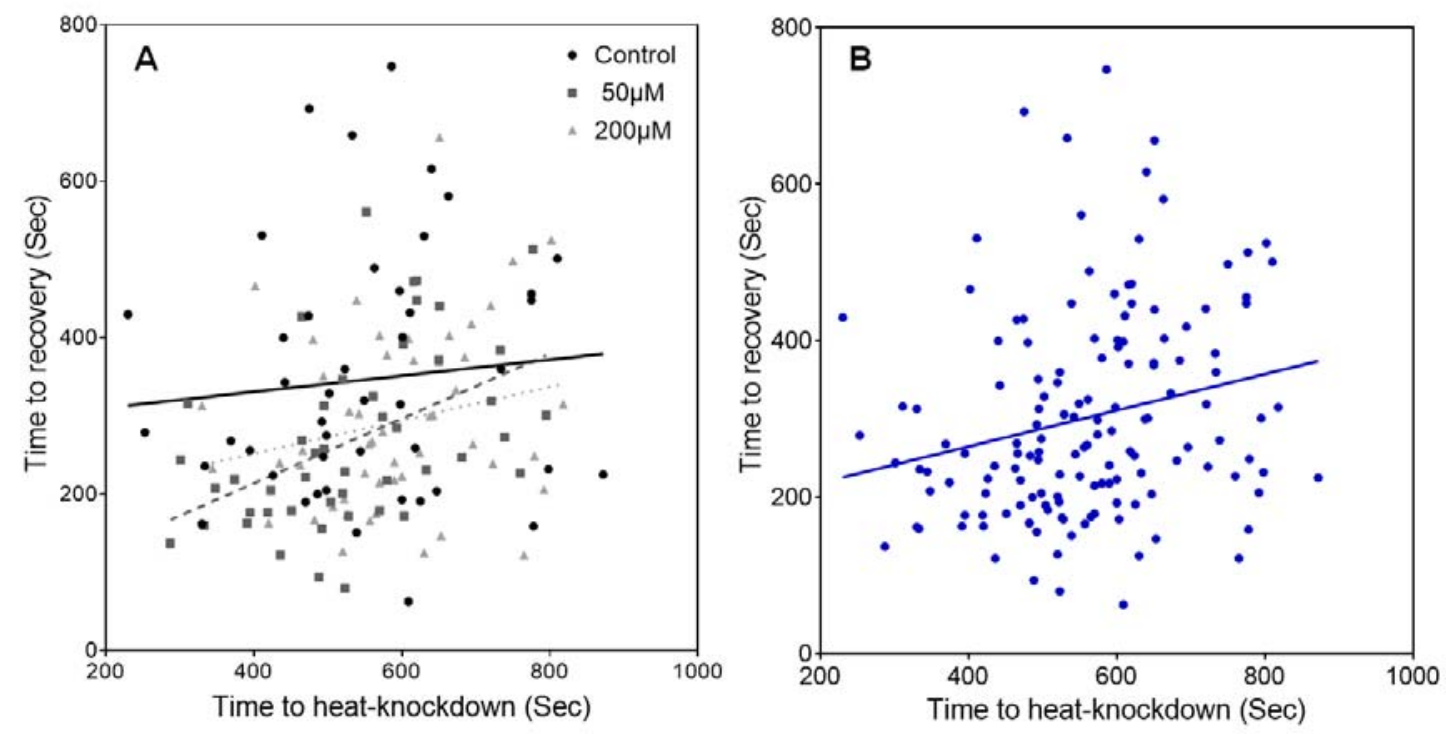

Figure 5. Time to recovery as a function of time to heat-knockdown at $41^{\circ} \mathrm{C}$ in flies. Fig. 5A: Linear regression for individuals pooled as a function of treatment. No significant correlation was observed, with exception of a weak linear relationship in $50 \mu \mathrm{M}$ rapamycin fed flies $\left(R^{2}=0.22, p<0.001\right.$; Table 1). Fig.5B: regression on the global dataset, a minor but statistically significant linear correlation was present $\left(R^{2}=0.05, p<0.01\right.$; Table 1$)$.

Table 1. Details of the linear regressions calculated for time to recovery as a function of time to heat-knockdown at $41^{\circ} \mathrm{C}$ in individual flies. A weak but statistically significant relationship was observed for flies fed rapamycin at $50 \mu \mathrm{M}$ and on the global dataset.

\begin{tabular}{|l|c|c|c|}
\multicolumn{1}{|c|}{ Condition } & \multicolumn{1}{c|}{ Equation } & $\mathbf{R}^{2}$ & $\boldsymbol{p}$ value \\
\hline Rapamycin $0 \mu \mathrm{M}$ (Control) & $\mathrm{Y}=0,1028^{\star} \mathrm{X}+289,9$ & 0.008 & 0.54 \\
\hline Rapamycin $50 \mu \mathrm{M}$ & $\mathrm{Y}=0,4141^{\star} \mathrm{X}+48,21$ & 0.220 & $9^{\star} 10^{-4}$ \\
\hline Rapamycin $200 \mu \mathrm{M}$ & $\mathrm{Y}=0,2135^{\star} \mathrm{X}+166,5$ & 0.044 & 0.13 \\
\hline Global dataset & $\mathrm{Y}=0,2315^{\star} \mathrm{X}+172,3$ & 0.051 & $6.3^{\star} 10^{-3}$ \\
\hline
\end{tabular}

\section{Discussion}


The inhibition of TOR as a result of rapamycin exposure in D. melanogaster is a welldocumented process (Lamming, 2016). First, in this species, rapamycin feeding increases the number of visible lysosomes and/or autolysosomes in the midgut stained with Lysotracker (Bjedov et al., 2009), which was also confirmed in our results (Fig. 1). This indicates intracellular absorption of the drug through feeding, and a probable local inhibition of TOR, triggering an increase of autophagic flux in exposed tissues. Second, rapamycin is known to reduce the downstream phosphorylation of p70 S6 kinase involved in ribosome biogenesis, and disrupts signaling of cell nutrient availability (Zhang et al., 2000). These effects likely account for an important part of the delayed Drosophila cellular growth and proliferation observed after rapamycin exposure (Zhang et al., 2000), which we also witnessed in our experiments through hindered larval development (Fig. 2). Taken together with the increased autolysosome formation in the gut, this indicates that rapamycin feeding does cause the expected systemic effects in link with TOR inhibition in our experimental system, and inducing autophagy at least locally in the gut.

Overall, autophagy's role in protecting from abiotic stressors such a desiccation, toxics, or direct oxidative damages in ectotherms has been highlighted numerous times (Moore et al., 2006, Bjedov et al., 2009; Minois et al., 2012; Teets and Denlinger, 2013; Mizunoe et al., 2018; Zhang et al., 2019; Babczyńska et al., 2020, Valko et al., 2021). In the specific case of thermal stress, further correlations between autophagy genes upregulation and cold acclimation have been made (Gerken et al., 2015; Des Marteaux et al., 2017; Tachibana et al., 2019). However, a causal link between autophagy induction, heat-acclimation and increased upper heat tolerance had never been proven until more recently in Caenorhabditis elegans (Kumsta et al., 2017). Our results first confirm that rapamycin treatment induces autophagy in flies (Fig.1), ant that this correlates with an increase in heat-tolerance (Fig. 3) also recently documented in $D$. melanogaster (Rohde et al., 2021). Second, we also show that $50 \mu \mathrm{M}$ is already sufficient to reach a plateau response under a $37^{\circ} \mathrm{C}$ stress exposure. Such delayed heat-knockdown was not observed in experiments conducted at $41^{\circ} \mathrm{C}$, which likely underpins that knockdowns occurred too fast in this condition for us to pick a statistically significant response (Fig. 4A). Third, we were able to detect a benefit of rapamycin exposure in reducing time to recovery post heat-knockdown (Fig. 4B). This indicates that autophagy induction could be involved in both basal heat-hardiness and in heat-stress recovery. And finally, we found little correlation by plotting individual flies' times for heat-knockdown at $41^{\circ} \mathrm{C}$ with their times to recovery (Fig. 5). This means that the heathardier flies (that took longer to lose their righting response), were not necessarily the ones to recover faster from heat knockdown, independently of treatments. This also suggest heattolerance and stress-recovery to be at least based on partly different set of mechanisms 
(Willot et al., 2021). Overall, our results thus further bridge the link between TOR inhibition, autophagy induction, and heat-tolerance in $D$. melanogaster.

Autophagy is known to articulate with other molecular level processes related to the stress response (Kroemer et al., 2010). Example of these mechanisms studies in insects have been for example the heat-shock proteins induction (Feder and Hoffmann, 1999,) the antioxidant response (Lalouette et al., 2011, Valko, 2021), up-regulation of proteasomal degradation pathways (Colinet et al., 2007; Kirstensen et al., 2016), modification of cellular membranes (Hazel, 1995; Overgaard et al., 2005; Koštál et al., 2013) and the mitochondrial function (Colinet et al., 2017; Havird et al., 2019; Chung and Schultze 2020). At the molecular level, TOR inhibition and autophagy upregulation as a result of rapamycin exposure is likely to promote protein homeostasis and clearance of toxic protein aggregates (Kumsta et al., 2017). In turn, enhanced protein homeostasis would explain the increased heat-tolerance and decreased recovery time from heat-knockdown we observed after exposing flies to rapamycin. However, the onset of heat-coma in D. melanogaster correlates with neural dysfunction (Jorgensen et al., 2020) and in this context, the mechanisms by which increased autophagy could prove beneficial to withstand heat-stress from a neurological perspective requires more careful exploration. In $C$. elegans, the full benefit of heat-hardening was indeed reliant, but only partially, on the expression of genes involved in several steps of the autophagy process (Kumsta et al., 2017). It would thus be particularly interesting to appreciate the exact contribution of autophagy induction in the context of thermal acclimation adopting a gene-based approach in the brain. Finally, exploring the relationship between heat-stress and modulation of chaperone-mediated autophagy (CMA) would be another relevant aspect to investigate. This mechanism, mediated by the molecular chaperone hsc70, directly guides proteins to be degraded to the lysosome (Cuervo and Wong, 2013). CMA thus represents a distinct protein degradation pathway from the proteasome, and could be proven of importance to increase protein turn-over and the context of heat-stress tolerance.

In conclusion, our results show that rapamycin exposure induces autophagy in $D$. melanogaster, which correlates with an increase in heat-tolerance and speed of recovery post-knockdown in adults. This highlights the induction of autophagy as potentially key mechanism to increase heat-tolerance in insects. A deeper exploration of autophagy's role in relation to other stress responsive pathways remains however necessary, and could provide important perspectives on the broader integrated cellular stress-response to temperatures in ectotherms. 


\section{Authors' contribution}

Quentin Willot: Conceptualization, Methodology, Investigation, Formal analysis, Writing original draft, Visualization. Andre du Toit: Methodology, Investigation. Ben Loos: Conceptualization, Methodology, Resources, Writing - review \& editing. John S. Terblanche: Conceptualization, Resources, Writing - review \& editing, Funding acquisition.

\section{Acknowledgment}

We thank Erika Huisamen for help with flies' husbandry as well as Lize Engelbrecht and the team from the Central Analytical Facility of Stellenbosch University for their help with the experimental setup.

\section{Competing interests}

We declare we have no competing interests.

\section{Funding}

This work was supported by a Claude Leon Foundation post-doctoral fellowship (to Q.W.) and a running cost funding (to J.T.) from the DSI-NRF Center of Excellence for Invasion Biology.

\section{References}

Babczyńska, A., Nowak, A., Kafel, A., Łozowski, B., Rost-Roszkowska, M., \& Tarnawska, M. et al. (2020). Autophagy: a necessary defense against extreme cadmium intoxication in a multigenerational 2D experiment. Scientific Reports, 10(1). doi: 10.1038/s41598-02078316-z

Bjedov, I., Toivonen, J., Kerr, F., Slack, C., Jacobson, J., Foley, A., \& Partridge, L. (2010). Mechanisms of Life Span Extension by Rapamycin in the Fruit Fly Drosophila melanogaster. Cell Metabolism, 11(1), 35-46. doi: 10.1016/j.cmet.2009.11.010

Chung, D., \& Schulte, P. (2020). Mitochondria and the thermal limits of ectotherms. The Journal Of Experimental Biology, 223(20), jeb227801. doi: 10.1242/jeb.227801

Colinet, H., Nguyen, T., Cloutier, C., Michaud, D., \& Hance, T. (2007). Proteomic profiling of a parasitic wasp exposed to constant and fluctuating cold exposure. Insect Biochemistry And Molecular Biology, 37(11), 1177-1188. doi: 10.1016/j.ibmb.2007.07.004 
Colinet, H., Renault, D., \& Roussel, D. (2017). Cold acclimation allows Drosophila flies to maintain mitochondrial functioning under cold stress. Insect Biochemistry And Molecular Biology, 80, 52-60. doi: 10.1016/j.ibmb.2016.11.007

Cuervo, A., \& Wong, E. (2013). Chaperone-mediated autophagy: roles in disease and aging. Cell Research, 24(1), 92-104. doi: 10.1038/cr.2013.153

Des Marteaux, L., McKinnon, A., Udaka, H., Toxopeus, J., \& Sinclair, B. (2017). Effects of cold-acclimation on gene expression in Fall field cricket (Gryllus pennsylvanicus) ionoregulatory tissues. BMC Genomics, 18(1). doi: 10.1186/s12864-017-3711-9

Feder, M., \& Hofmann, G. (1999). HEAT-SHOCK PROTEINS, MOLECULAR CHAPERONES, AND THE STRESS RESPONSE: Evolutionary and Ecological Physiology. Annual Review Of Physiology, 61(1), 243-282. doi: 10.1146/annurev.physiol.61.1.243

Gerken, A., Eller, O., Hahn, D., \& Morgan, T. (2015). Constraints, independence, and evolution of thermal plasticity: Probing genetic architecture of long- and short-term thermal acclimation. Proceedings Of The National Academy Of Sciences, 112(14), 4399-4404. doi: $10.1073 /$ pnas. 1503456112

González-Tokman, D., Córdoba-Aguilar, A., Dáttilo, W., Lira-Noriega, A., Sánchez-Guillén, R., \& Villalobos, F. (2020). Insect responses to heat: physiological mechanisms, evolution and ecological implications in a warming world. Biological Reviews, 95(3), 802-821. doi: $10.1111 /$ brv. 12588

Havird, J., Shah, A., \& Chicco, A. (2019). Powerhouses in the cold: mitochondrial function during thermal acclimation in montane mayflies. Philosophical Transactions Of The Royal Society B: Biological Sciences, 375(1790), 20190181. doi: 10.1098/rstb.2019.0181

Hazel, J. (1995). Thermal Adaptation in Biological Membranes: Is Homeoviscous Adaptation the Explanation?. Annual Review Of Physiology, 57(1), 19-42. doi: 10.1146/annurev.ph.57.030195.000315

Jørgensen, L. B., Robertson, R. M., \& Overgaard, J. (2020). Neural dysfunction correlates with heat coma and CTmax in Drosophila but does not set the boundaries for heat stress survival. Journal of Experimental Biology. doi:10.1242/jeb.218750 
Koštál, V., Urban, T., Řimnáčová, L., Berková, P., \& Šimek, P. (2013). Seasonal changes in minor membrane phospholipid classes, sterols and tocopherols in overwintering insect, Pyrrhocoris apterus. Journal Of Insect Physiology, 59(9), 934-941. doi: 10.1016/j.jinsphys.2013.06.008

Kristensen, T., Kjeldal, H., Schou, M., \& Nielsen, J. (2016). Proteomic data reveal a physiological basis for costs and benefits associated with thermal acclimation. The Journal Of Experimental Biology, 219(7), 969-976. doi: 10.1242/jeb.132696

Kroemer, G., Mariño, G., \& Levine, B. (2010). Autophagy and the Integrated Stress Response. Molecular Cell, 40(2), 280-293. doi: 10.1016/j.molcel.2010.09.023

Kumsta, C., Chang, J., Schmalz, J., \& Hansen, M. (2017). Hormetic heat stress and HSF-1 induce autophagy to improve survival and proteostasis in C. elegans. Nature Communications, 8(1). doi: 10.1038/ncomms 14337

Lamming, D. (2016). Inhibition of the Mechanistic Target of Rapamycin (mTOR)-Rapamycin and Beyond. Cold Spring Harbor Perspectives In Medicine, 6(5), a025924. doi: 10.1101/cshperspect.a025924

Lalouette, L., Williams, C., Hervant, F., Sinclair, B., \& Renault, D. (2011). Metabolic rate and oxidative stress in insects exposed to low temperature thermal fluctuations. Comparative Biochemistry And Physiology Part A: Molecular \& Integrative Physiology, 158(2), 229-234. doi: 10.1016/j.cbpa.2010.11.007

Lewis, E. B. (1960). A new standard food medium. Drosophila Information Service, 34(117), $1-55$.

Lindquist, S. (1986). The Heat-Shock Response. Annual Review Of Biochemistry, 55(1), 1151-1191. doi: 10.1146/annurev.bi.55.070186.005443

Markow, T. A., \& O'Grady, P. (2005). Drosophila: a guide to species identification and use. Elsevier.

Maruzs, T., Simon-Vecsei, Z., Kiss, V., Csizmadia, T., \& Juhász, G. (2019). On the Fly: Recent Progress on Autophagy and Aging in Drosophila. Frontiers in Cell and Developmental Biology, 7. doi:10.3389/fcell.2019.00140 
Minois, N., Carmona-Gutierrez, D., Bauer, M., Rockenfeller, P., Eisenberg, T., \& Brandhorst, S. et al. (2012). Spermidine promotes stress resistance in Drosophila melanogaster through autophagy-dependent and -independent pathways. Cell Death \& Disease, 3(10), e401-e401. doi: 10.1038/cddis.2012.139

Mizunoe, Y., Kobayashi, M., Sudo, Y., Watanabe, S., Yasukawa, H., \& Natori, D. et al. (2018). Trehalose protects against oxidative stress by regulating the Keap1-Nrf2 and autophagy pathways. Redox Biology, 15, 115-124. doi: 10.1016/j.redox.2017.09.007

Mizushima, N. (2007). Autophagy: process and function. Genes \& Development, 21(22), 2861-2873. doi: 10.1101/gad.1599207

Moore, M., Allen, J., \& Somerfield, P. (2006). Autophagy: Role in surviving environmental stress. Marine Environmental Research, 62, S420-S425. doi: 10.1016/j.marenvres.2006.04.055

Overgaard, J., Sørensen, J., Petersen, S., Loeschcke, V., \& Holmstrup, M. (2005). Changes in membrane lipid composition following rapid cold hardening in Drosophila melanogaster. Journal Of Insect Physiology, 51(11), 1173-1182. doi: 10.1016/j.jinsphys.2005.06.007

Pirkkala, L., Nykänen, P., \& Sistonen, L. (2001). Roles of the heat shock transcription factors in regulation of the heat shock response and beyond. The FASEB Journal, 15(7), 11181131. doi: 10.1096/fj00-0294rev

Richter, K., Haslbeck, M., \& Buchner, J. (2010). The Heat Shock Response: Life on the Verge of Death. Molecular Cell, 40(2), 253-266. doi: 10.1016/j.molcel.2010.10.006

Rohde, P. D., Bøcker, A., Jensen, C. A. B., Bergstrøm, A. L., Madsen, M. I. J., Christensen, S. L., ... Kristensen, T. N. (2021). Genotype and Trait Specific Responses to Rapamycin Intake in Drosophila melanogaster. Insects, 12(5), 474. doi:10.3390/insects12050474

Schneider, C., Rasband, W., \& Eliceiri, K. (2012). NIH Image to ImageJ: 25 years of image analysis. Nature Methods, 9(7), 671-675. doi: 10.1038/nmeth.2089

Somero, G. (2020). The cellular stress response and temperature: Function, regulation, and evolution. Journal Of Experimental Zoology Part A: Ecological And Integrative Physiology, 333(6), 379-397. doi: 10.1002/jez.2344 
Teets, N., \& Denlinger, D. (2013). Autophagy in Antarctica. Autophagy, 9(4), 629-631. doi: 10.4161/auto.23643

Valko, A., Perez-Pandolfo, S., Sorianello, E., Brech, A., Wappner, P., \& Melani, M. (2021). Adaptation to hypoxia in Drosophila melanogaster requires autophagy. Autophagy, 1-12. doi: $10.1080 / 15548627.2021 .1991191$

Willot, Q., Loos, B., \& Terblanche, J. S. (2021). Interactions between developmental and adult acclimation have distinct consequences for heat tolerance and heat stress recovery. Journal of Experimental Biology, 224(16). doi:10.1242/jeb.242479

Wullschleger, S., Loewith, R., \& Hall, M. (2006). TOR Signaling in Growth and Metabolism. Cell, 124(3), 471-484. doi: 10.1016/j.cell.2006.01.016

Zhang, H. (2000). Regulation of cellular growth by the Drosophila target of rapamycin dTOR. Genes \& Development, 14(21), 2712-2724. doi: 10.1101/gad.835000

Zhang, D., Xiao, Z., Zeng, B., Li, K., \& Tang, Y. (2019). Insect Behavior and Physiological Adaptation Mechanisms Under Starvation Stress. Frontiers In Physiology, 10. doi: 10.3389/fphys.2019.00163

Zhu, J., Hannan, S. B., Dräger, N. M., Vereshchagina, N., Krahl, A.-C., Fu, Y., ... Rasse, T. M. (2021). Autophagy inhibition rescues structural and functional defects caused by the loss of mitochondrial chaperone Hsc70-5 in Drosophila. Autophagy, 17(10), 3160-3174. doi:10.1080/15548627.2020.1871211 